\title{
The Effect of Extending the Length of the Coupling Coils in a Muon Ionization Cooling Channel
}

\author{
Michael A. Green \\ Lawrence Berkeley National Laboratory \\ Berkeley CA 94720, USA
}

\begin{abstract}
RF cavities are used to re-accelerate muons that have been cooled by absorbers that are in low beta regions of a muon ionization cooling channel. A superconducting coupling magnet (or magnets) are around or among the RF cavities of a muon ionization-cooling channel. The field from the magnet guides the muons so that they are kept within the iris of the RF cavities that are used to accelerate the muons. This report compares the use of a single short coupling magnet with an extended coupling magnet that has one or more superconducting coils as part of a muon-cooling channel of the same design as the muon ionization cooling experiment (MICE). Whether the superconducting magnet is short and thick or long and this affects the magnet stored energy and the peak field in the winding. The magnetic field distribution also affects is the muon beam optics in the cooling cell of a muon cooling channel.
\end{abstract}

Keywords: Muon Ionization Cooling, Superconducting Magnet.

PACS: $14.60 \mathrm{ef}, 84.71 \mathrm{ba}$

\section{INTRODUCTION}

Muon ionization cooling [1] in a linear channel requires; 1) low $\mathrm{Z}$ absorbers to reduce the longitudinal and transverse momentum from the muons being cooled, 2) RF cavities to re-accelerate the muons so that the longitudinal momentum is recovered, and 3) a magnetic field that guides and focuses the muons in the cooling channel. A muon-cooling channel will have two types of superconducting magnets within it. The first type of magnet is the focusing magnet, which will focus the muon beam into a low beta region where muon ionization cooling can take place. The second type of magnet is the coupling magnet that guides the muon beam in the RF cavities that are between the absorbers. This report deals with the second type of superconducting magnet.

The muon ionization cooling experiment [2] (MICE) under construction at the Rutherford Appleton Laboratory in the United Kingdom will demonstrate muon cooling with one cell of cooling in a lattice that is similar to the lattice described in the level II study of a neutrino factory [3]. The muon cooling channel in the level II study has cells that consist of two focusing magnets and absorber and two sets of RF cavities with their coupling magnets that keep the muon bean in the cavities while it is being reaccelerated. The lattice used for the level II study and MICE may operate with the focusing magnets in the flip mode (the field changes polarity as one goes through the focusing magnet) or the non-flip mode (the magnetic field polarity doesn't change) [4]. Both modes will produce a region of low beam beta, which improves muon ionization cooling.

The coupling coils for MICE are large radius coils that go around the 201.25 MHz RF cavities [5]. These coils have a large inner radius $>0.7 \mathrm{~m}$ (dictated by the physical size of the RF cavities) and large currents $>3.3$ MA. The length of the MICE coupling coil is limited by the spacing between the RF couplers for the two center MICE cavities. The result is a coupling coil with a large stored energy and a non-optimal use for the current that is carried within the coil.

During the level II study, a small-radius coupling coil around the beam pipe between the center cavities was studied. A single small-radius coupling coil did not prevent muons from being lost on the irises of the $\mathrm{RF}$ cavities that were away from the magnet. A large single coupling coil was adopted for both the level II study lattice and the MICE lattice, because the muon beam would stay constrained within the irises of the four RF cavities that are between the absorbers.

A three-dimensional view of the MICE magnets is shown in FIGURE 1. The two coupling coils are the large coils shown in FIGURE 1. The focusing magnets are pairs of smaller coils on either side of the coupling coils. The five-coil tracker magnets are at the two ends of the MICE channel. 


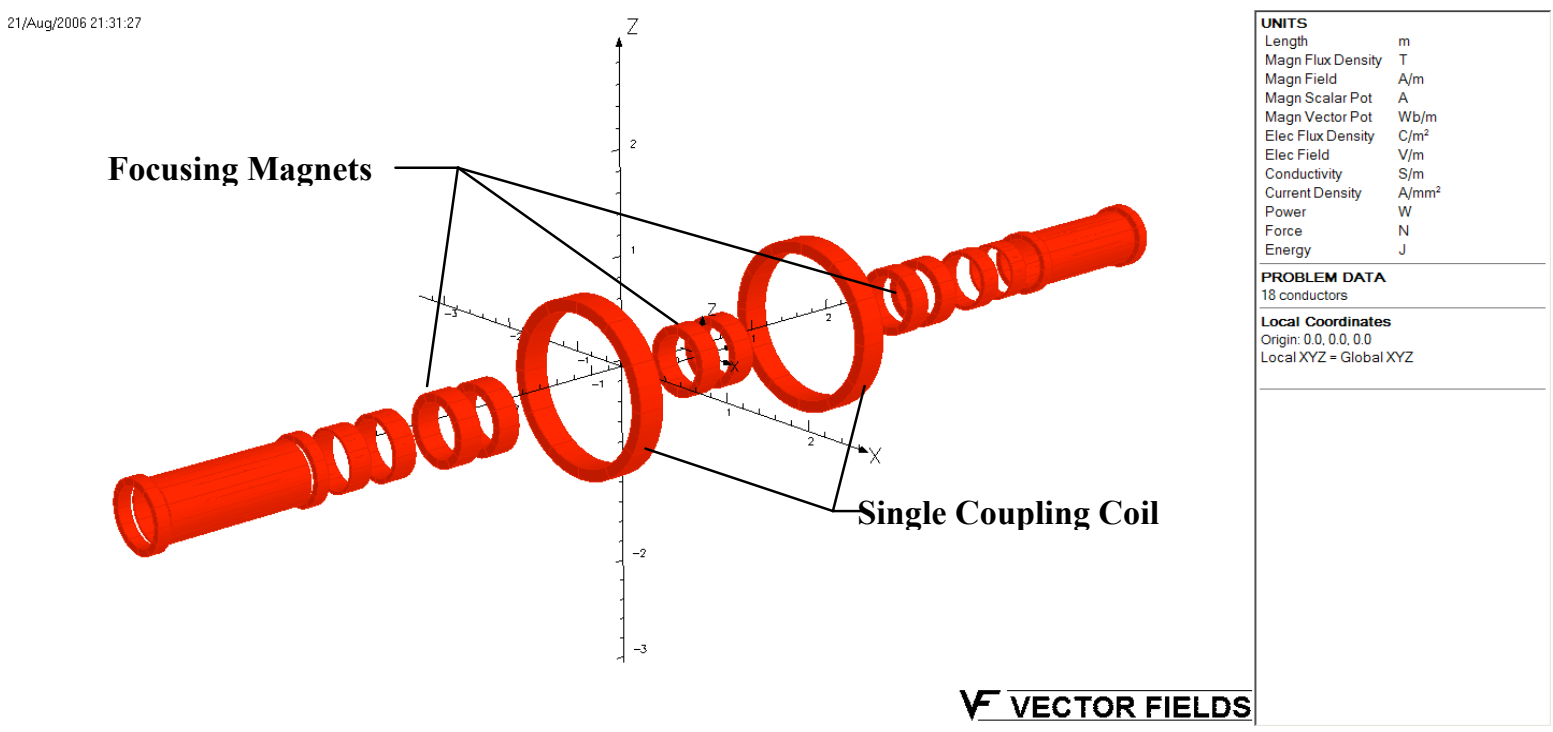

FIGURE 1. A Three-dimensional View of the MICE Coils with a Single Short Large-radius Coupling Coil per Half-cell.

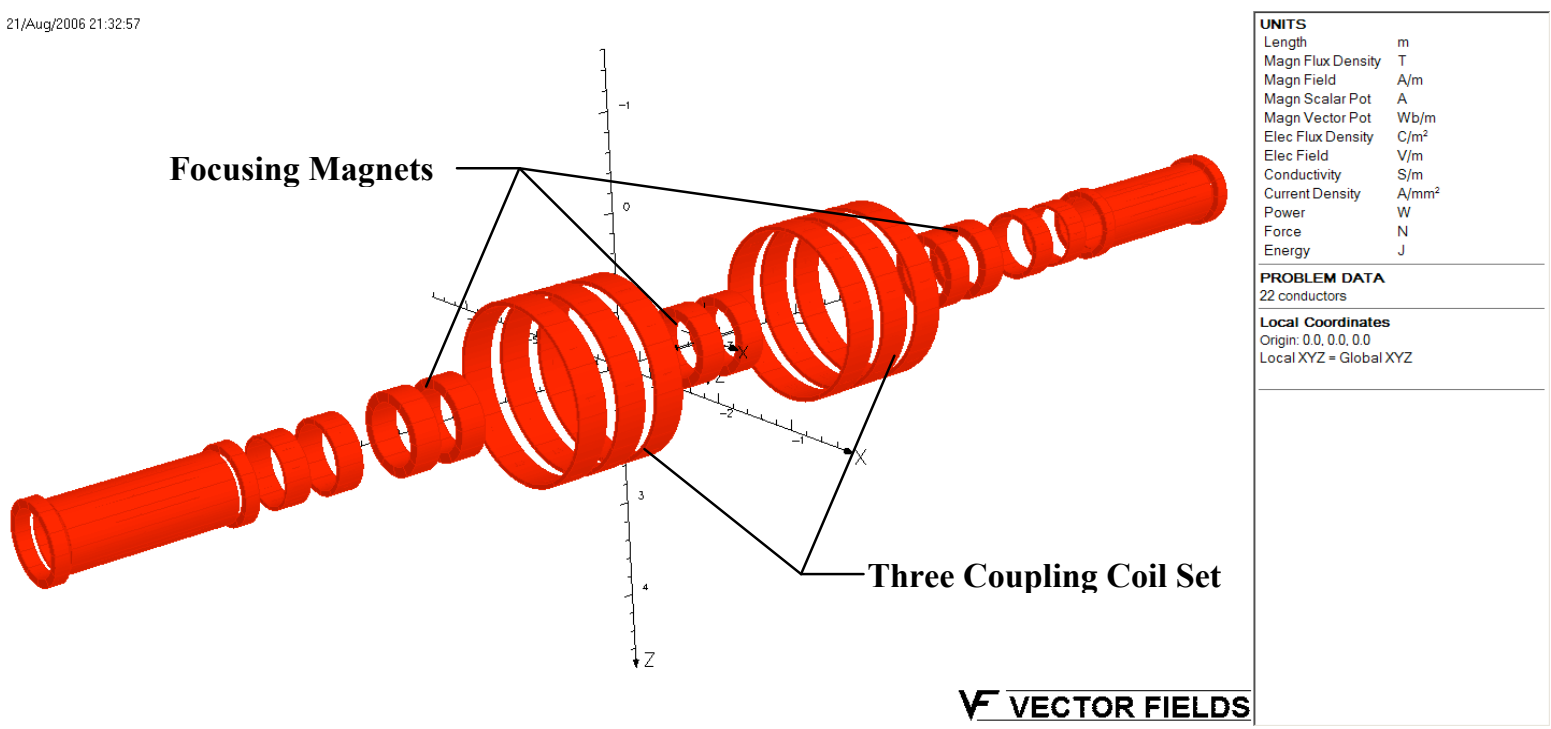

FIGURE 2. A Three-dimensional View of the MICE Coils with Three Short Large-radius Coupling Coils per Half-cell.

\section{SHORT AND LONG COUPLING COILS}

The MICE lattice was used to study the effect of extending the length of the coupling coil. The extended coupling coil used for the study is a set of three coils (see FIGURE 2). Each coil has the same length as the original coupling coil. The new coupling coils have one-third the thickness of the original coupling coil. The inner radius of the three coils is the same as the original coupling coil. The three coils are spaced so that they are equivalent to a single coil that is 1150 -mm long, with an ID of $725 \mathrm{~mm}$.
FIGURE 3 shows a cross-section of the three coils with enough space between them so that RF couplers that are $100 \mathrm{~mm}$ in diameter can be brought out through the magnet. The total current in the three coils shown in FIGURE 2 is the same as the total current in the short coupling coil shown in FIGURE 1.

The three coupling coils shown in FIGURE 2 and FIGURE 3 will behave magnetically as a single coil that is $1150 \mathrm{~mm}$ long. (This long coil has the same ampere-turns as the single coupling coil that is shown in FIGURE 1.) Even though the coupling coils shown in FIGURES 2 and 3 have the same number of ampere-turns as the coil in FIGURE 1, the magnetic field distribution on and off axis is different. 


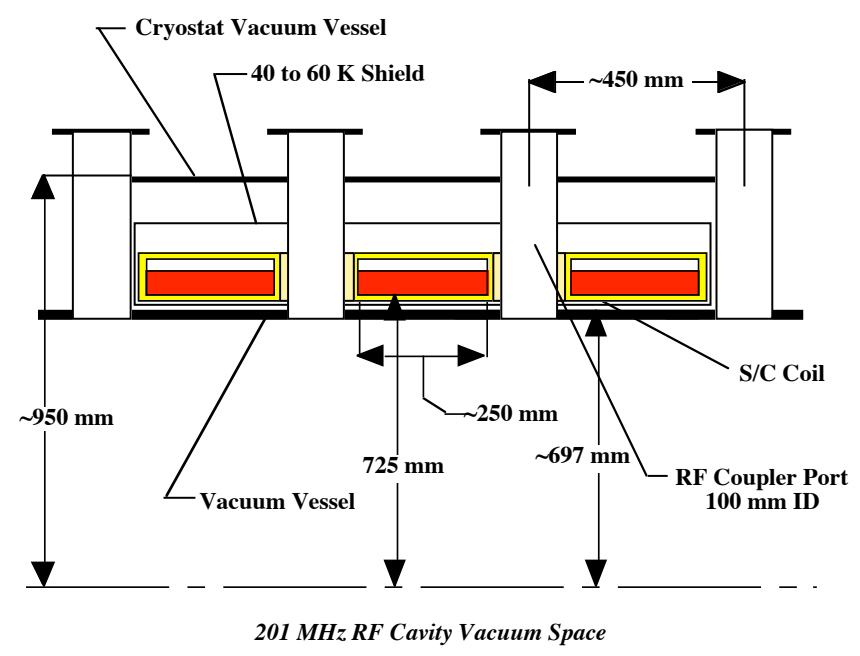

FIGURE 3. A cross-section of the Three-coil Set that Represents a Single Coupling Coil that is 1150-mm Long.

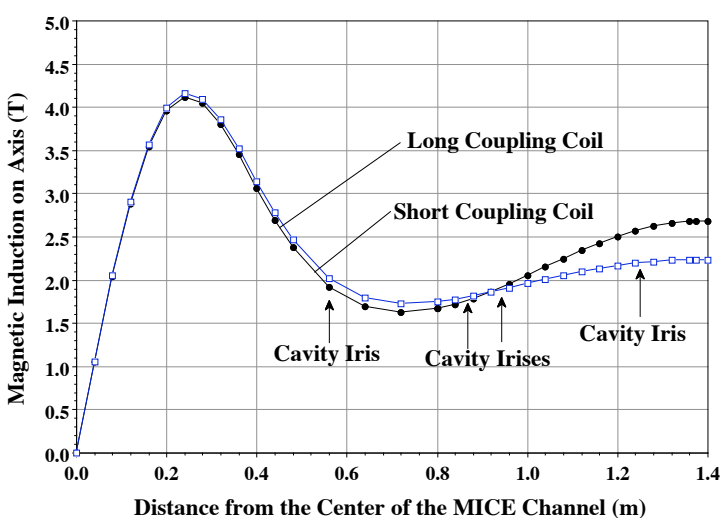

FIGURE 4. The On-axis Magnetic Field as a Function of the Distance from the Center of the Focusing Magnet for a Single Coupling Coil shown in FIGURE 1 and an Extended Coupling Coil represented by the Three Coils shown in FIGURES 2 and 3. (Note: the center of the RF cavity coupling coil module is at $\mathrm{z}= \pm 1.375 \mathrm{~m}$.)

FIGURE 4 shows the on-axis magnetic induction as a function of the distance from the center of MICE (defined as the center of the center focusing magnet in the MICE channel). The magnetic field direction on axis in MICE is only in the $\mathrm{z}$ direction. The magnetic field plot shown in FIGURE 4 applies for MICE operating in the flip mode with an average momentum of $240 \mathrm{MeV} / \mathrm{c}$. The beta at the center of the focusing magnet (at $\mathrm{z}=0$ ) is different for the two cases.

Table 1 compares the magnet parameters for the single-coil coupling magnet shown in FIGURE 1 with the three-coil coupling magnet shown in FIGURE 2 and FIGURE 3. In both cases the inner coil radius was assumed to be $725 \mathrm{~mm}$. The total length of the three coils is $750 \mathrm{~mm}$ versus $250 \mathrm{~mm}$ for the single coil. The ampere-turns are the same for both magnets.
TABLE 1. A Comparison of the Single Coil Coupling Magnet with a Three coil Extended Coupling Magnet.

\begin{tabular}{lcc}
\hline \multicolumn{1}{c}{ Magnet Parameter } & 1 Coil & 3 Coil \\
\hline Number of Magnet Coils & 1 & 3 \\
Magnet Coil Length $(\mathrm{mm})$ & 250 & 250 \\
Spacing between Coils $(\mathrm{mm})$ & $\mathrm{NA}$ & 200 \\
Equivalent Magnet Length $(\mathrm{mm})$ & 250 & 1150 \\
Inner Radius of the Coil $(\mathrm{mm})$ & 725 & 725 \\
Coil Thickness $(\mathrm{mm})$ & 114.4 & 37.4 \\
Number of Layers per Coil & 104 & 35 \\
Number of Turns per Layer & 151 & 151 \\
Magnet Self Inductance $(\mathrm{H})$ & $\sim 563$ & $\sim 310$ \\
Maximum Magnet Current $\mathrm{I}_{\mathrm{M}}(\mathrm{A})$ & 213.2 & 211.2 \\
Magnet Stored Energy at $\mathrm{I}_{\mathrm{M}}(\mathrm{MJ})$ & $\sim 12.8$ & $\sim 6.9$ \\
Number of Quench Circuits & 8 & 3 \\
Maximum Quench Voltage at $\mathrm{I}_{\mathrm{M}}(\mathrm{kV})$ & $\sim 1.4$ & $\sim 2.1$ \\
Layer-to-layer Voltage at $\mathrm{I}_{\mathrm{M}}(\mathrm{V})$ & $\sim 220$ & $\sim 120$ \\
Peak Induction in the Coil at $\mathrm{I}_{\mathrm{M}}(\mathrm{T})$ & 7.81 & 3.58 \\
Temp Margin at $\mathrm{I}_{\mathrm{M}}$ \& 4.2 K $(\mathrm{K})$ & $\sim 0.6$ & $\sim 2.6$ \\
\hline
\end{tabular}

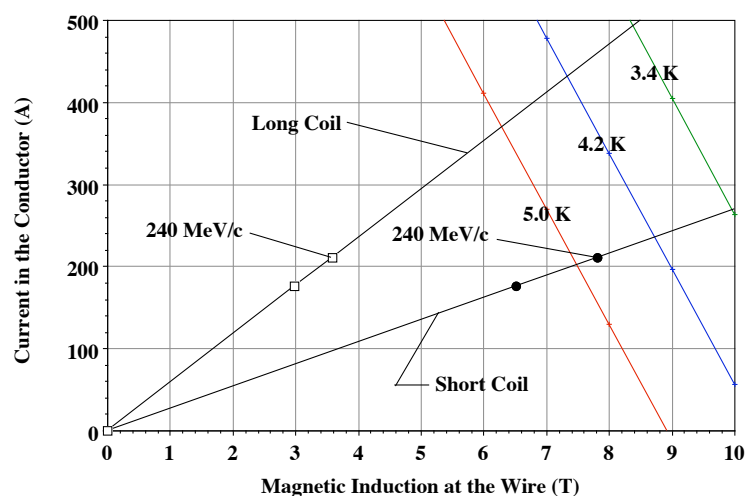

FIGURE 5. The Coupling Magnet Load Lines for the Single Short Coil and the Extended Three Coil Magnet and the $\mathrm{I}_{\mathrm{C}}$ of the Superconductor at $3.4 \mathrm{~K}, 4.2 \mathrm{~K}$ and $5.0 \mathrm{~K}$. The maximum magnet current is for $\mathrm{p}=240 \mathrm{MeV} / \mathrm{c}$. 
From TABLE 1 and FIGURE 5, it is clear that extending the length of the coupling coil in a lattice that is similar to the MICE lattice will decrease the magnet self inductance and stored energy. The peak magnetic field at the winding will go down. The two phenomena are directly connected with one another. A short thick coil of a given current density has a higher self-field than a long thin coil.

When one looks at the field on axis in FIGURE 4 for the short coil, the peak on axis induction due to the coupling coil alone is $\sim 2.7 \mathrm{~T}$. The peak induction in the short coil is $\sim 7.8 \mathrm{~T}$. The difference between the on axis induction and the peak induction is $\sim 5.1 \mathrm{~T}$. This difference is due to the self-field around the coil. When one looks at the extended coupling coil the peak induction this peak on axis induction drops to about $\sim 2.2 \mathrm{~T}$. The peak induction in the coil is $3.6 \mathrm{~T}$. The difference between the peak on axis induction and the peak induction is $\sim 1.3 \mathrm{~T}$. Much of the short coil stored energy is stored around the coil.

As illustrated in FIGURE 5, increased temperature margin for the extended coil is due to the fact that the peak magnetic field at the conductor is lower. Thus the extended coil uses superconductor more efficiently than does the short coupling coil. In general, a magnet with a lower stored energy is less expensive to build. Whether a lower stored energy magnet is cheaper to build depends on the details of the design of the magnet and its cryostat.

The magnetic induction on the outside of the magnet is also lower for the extended (three coil) coupling magnet. A plot of magnetic induction versus radius at $\mathrm{z}=1.375 \mathrm{~m}$ is shown in FIGURE 6 . This plot shows that the magnet field outside of the long coupling coil is much lower than the field at the same radius for a short coupling coil. The field on the outside of the magnet affects HTS current leads [6] and the $4.2 \mathrm{~K}$ coolers [7] that might be used to cool the coupling magnet.

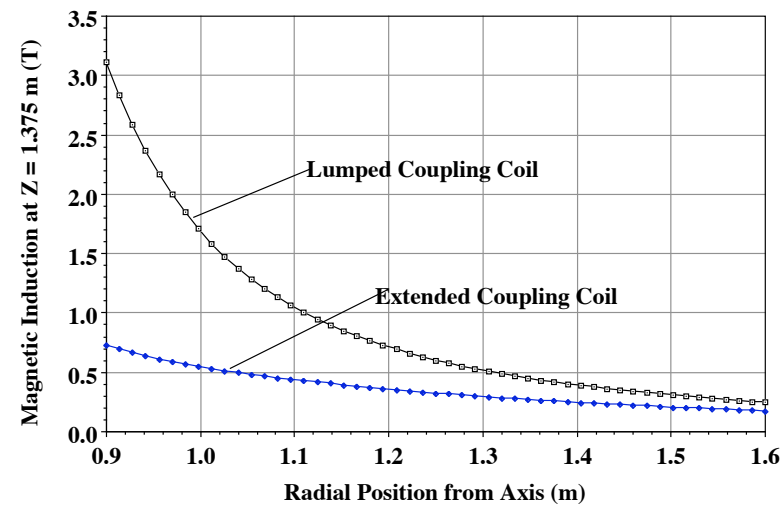

FIGURE 6. The magnetic Induction on the outside of short and long coupling magnets as a function of radius in the middle of the RF cavity space at $\mathrm{z}=1.375 \mathrm{~m}$.
For an experiment such as MICE, superconductor represents fifteen to twenty percent of the cost of the coupling coil. Other factors such as a complicated cryostat may affect the extended magnet cost more than the reduced cost of the conductor. As a result of this study, the length of the MICE coupling coil was increased from $250 \mathrm{~mm}$ to $285 \mathrm{~mm}$ [8]. Even though the MICE coupling magnet inner coil radius was increased from $725 \mathrm{~mm}$ to $750 \mathrm{~mm}$ (to reduce cryostat heat leak), the coupling magnet stored energy did not change. However, the magnet temperature margin did increase by about $0.2 \mathrm{~K}$. The increase in the temperature margin is due to the fact that the peak field in the coil went down from $\sim 7.8 \mathrm{~T}$ to $\sim 7.4 \mathrm{~T}$.

The change in the MICE coupling coil length resulted in the cryostat indents shown in FIGURE 7. The cryostat indents shown in the FIGURE 7 is allow the cryostat to fit around the RF coupler tubes and the $\mathrm{RF}$ vacuum ports. A change in the design of the MICE coupling coil beyond lengthening the magnet coil by $35 \mathrm{~mm}$ did not make sense, because a number of components of MICE had to be re-engineered. In some cases, existing components had to be rebuilt at increased cost to the experiment. In addition, a cost savings could be achieved by making the MICE and MUCOOL coupling coils with the same design.

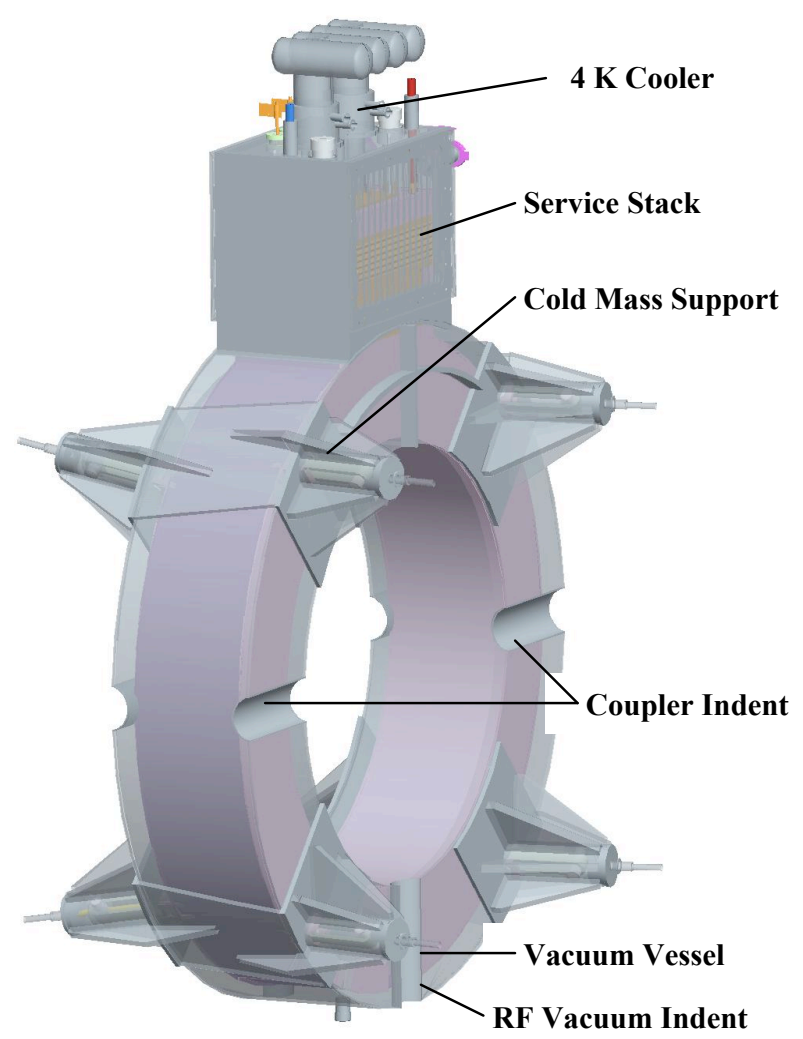

FIGURE 7. A 3-dimensional View of the Coupling Magnet Cryostat for MICE and MUCOOL. 


\section{THE EFFECT OF COUPLING COIL LENGTH ON THE TUNING OF THE MICE COOLING CHANNEL}

The field at the center of the RF cavities is lower for the extended coupling coil case as compared to the short coupling coil case. The magnet field increases as one moves toward the absorber focus coil (AFC) module (the focusing magnet). When the MICE channel was re-tuned using the tuning coils M1 and M2 in the tracker module, the beam beta at the center of the focusing magnet and absorber went down from $420 \mathrm{~mm}$ to $310 \mathrm{~mm}$. The decrease in the beam beta at the center of the AFC module results in an increase in the beam beta in the center of the RF cavity string and within the tuning coils for the tracker module. This effect is clearly seen in FIGURE 8.

Since the beam beta has increased at the irises of the RF cavities, it is likely that some off momentum particles will be lost on the RF cavity irises. As with the low beta cases in the original MICE lattice with the short coupling coil, the momentum acceptance is likely to go down as the beam beta within the AFC module absorber goes down.

One can increase the beam beta in the middle of the absorber by reducing the current in the focusing coil. In order to increase the beam beta back to the original design value $(420 \mathrm{~mm})$ for the MICE cooling channel, one must reduce the focusing magnet current of the order of 15 percent [4]. As a result, the temperature margin in the focusing magnet increases from $0.8 \mathrm{~K}$ to about $1.4 \mathrm{~K}$ (at a momentum of $240 \mathrm{MeV} / \mathrm{c}$ ). In order to re-tune the MICE with the long coupling coil, the number of ampere-turns in the coupling coil must go up of the order of 15 percent. Since the long coupling coil has a very large temperature margin, the coupling coil current can be increased 15 percent by reducing the temperature margin from $2.6 \mathrm{~K}$ to about $1.8 \mathrm{~K}$.

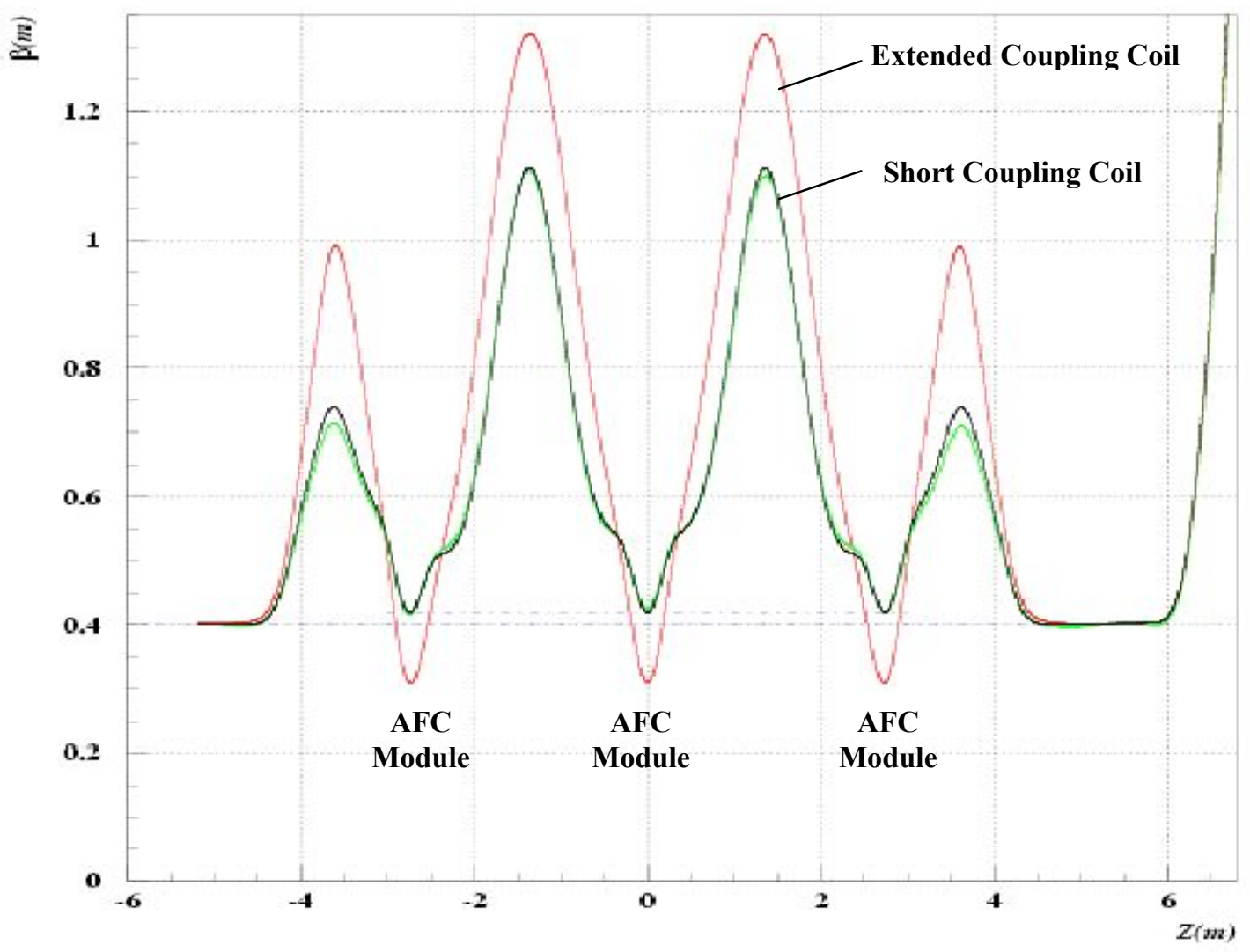

FIGURE 8. The MICE Channel Beta Function for an Optimized MICE Cooling Channel with a Short Coupling Coil and an Extended Coupling coil. The coil coupling coil current are the same as given in TABLE 1 . The channel was tuned using the tuning coils M1 and M2 in the spectrometer magnets. Note: the extended coupling coil results in a lower beta in the middle of the AFC module focusing magnet and a larger beta in the RF cavities and the tuning coils of the tracker module. The beam beta in the trackers remains the same for both the short coupling coil and the extended coupling coil. 


\section{MAGNETS BETWEEN THE RF CAVITIES AND OTHER LATTICES}

It has been proposed that the superconducting coupling magnets should be between the RF cavities instead of around the RF cavities. This might be a desirable in order to reduce cavity voltage breakdown, because the field would be in a small region within the cavity. Unfortunately, the region that would be in the magnetic field would be the region where the acceleration gradient is highest.

The key issue of having the magnets between the cavities is the size of the gap between the coils compared to the average radius of the superconducting coil. The gap between the coil ends should be as small as possible. If the gap between the ends of the superconducting coils is equal to the coil average radius, the peak field in the magnet coil will be roughly 1.5 times the minimum field along the axis of the channel through the cavity. If the gap between the coils is equal to twice the average radius of the coil, the peak field in the magnet will be roughly 3.2 times the minimum field in the cavity gap on axis. The ratios are a little worse if the coils are long compared to their average radius. Studies of induction linac magnets suggest that breaking the coils into pieces results in a better quality field and potentially better beam properties as well [9].

If the iris of a cavity has a radius of $210 \mathrm{~mm}$, the superconducting coil average radius could be as large as $300 \mathrm{~mm}$. At this radius, one could have a coil gap as large $400 \mathrm{~mm}$ and still have a reasonable ratio between the peak field in the magnet and the minimum field on axis field within the cavity gap. Breaking up the coil into lengths up to $600 \mathrm{~mm}$ appears to be desirable from a magnetic field standpoint.

It is interesting to contemplate a linear channel where the absorber is hydrogen gas at an average temperature of $90 \mathrm{~K}$ and a pressure of $20 \mathrm{bar}$. If the cavity acceleration gradient is $30 \mathrm{MV}$ per meter, the absorption length would have to be about 6.25 times the acceleration gap including hydrogen that is in the cavities. This suggests that for every meter of cavity gap, there would be an additional 5.25 meters of hydrogen, most of which is in a magnetic guide field.

\section{CONCLUDING COMMENTS}

The calculations presented in this report show that there are magnetic advantages in having an extended coupling coil around the RF cavities in a cooling channel as compared to a short thick coil. The short coil has a larger stored energy and a higher peak field in the winding. For a given superconductor volume, the short coil will have a lower temperature margin. It is reasonable to put gaps between the coils, if these gaps are needed for RF coupling, vacuum pumping and tuning. The gaps between the coils should be smaller than the coil radius.

Small diameter coils between RF cavities are an option provided the gap between the coils is less than the coil radius. If cold pressurized hydrogen gas is used as an absorber instead of liquid hydrogen, the magnet coil length will be several times longer than the accelerating gaps in the RF cavities. Breaking long coils into shorter lengths with gaps between the coils will improve the field quality in the magnet.

\section{ACKNOWLEDGEMENTS}

The author acknowledges the efforts of Soren Prestemon of the Lawrence Berkeley Laboratory for the field calculations on long and short coupling coils. The author acknowledges the efforts Marco Apollonio of Oxford University for his re-optimization of the MICE channel with extended coupling coils. This work was supported by the Office of Science, United States Department of Energy, under DOE contract DEAC02-05CH11231.

\section{REFERENCES}

1. N. Holtkamp and D. Finley eds. "A Feasibility Study of a Neutrino Source based on a Muon Storage Ring," Fermilab-Pub-00/108E, (2000)

2. G. Gregoire, G. Ryckewaert, L. Chevalier, et al, "MICE and International Muon Ionization Cooling Experiment Technical Reference Document," http://hep04.phys.itt.edu/cooldemo

3. S. Ozaki, R. B. Palmer, M. S. Zisman and J. C. Gallardo eds., "Feasibility Study II of a Muon-based Neutrino Source," BNL-52623, June 2001

2. Ullise Bravar, "Coil Currents in MICE," MCE Note 88 http://hep04.phys.itt.edu/cooldemo

5. M. A. Green, S. Q. Yang, U. Bravar, et al, "The Mechanical and Thermal Design for the MICE Coupling Solenoid Magnet," IEEE Transactions on Applied Superconductivity 15, No. 2, p 1279, (2005)

6. M. A. Green and H. Witte, "Using High Temperature Superconducting leads in a Magnetic Field," to be published in Advances in Cryogenic Engineering 53, AIP Press, Melville NY (2008)

7. M. A. Green and H. Witte, "The Use of Small Coolers in a Magnetic Field," to be published in Advances in Cryogenic Engineering 53, AIP Press, Melville NY (2008)

8. L. Wang, M. A. green, X. F. Xu, H. Wu, L. K. Li, X. L Guo, et al, "The Engineering of the $1.5 \mathrm{~m}$ Diameter Solenoid for the MICE RFCC Module," to be published in IEEE Transactions on Applied Superconductivity 18, No 2 (2008)

9. M. A. Green and R. J. Weggel, "Superconducting Magnets for Muon Capture and Phase Rotation," Proceedings of the Acc App-99 Conference, Long Beach CA, November 199 


\section{DISCLAIMER}

This document was prepared as an account of work sponsored by the United States Government. While this document is believed to contain correct information, neither the United States Government nor any agency thereof, nor The Regents of the University of California, nor any of their employees, makes any warranty, express or implied, or assumes any legal responsibility for the accuracy, completeness, or usefulness of any information, apparatus, product, or process disclosed, or represents that its use would not infringe privately owned rights. Reference herein to any specific commercial product, process, or service by its trade name, trademark, manufacturer, or otherwise, does not necessarily constitute or imply its endorsement, recommendation, or favoring by the United States Government or any agency thereof, or The Regents of the University of California. The views and opinions of authors expressed herein do not necessarily state or reflect those of the United States Government or any agency thereof, or The Regents of the University of California. 